\title{
The Baby Check booklet did not affect frequency of health service use in infants $\leq 6$ months of age
}

Thomson H, Ross S, Wilson P, et al Randomised controlled trial of effect of Baby Check on use of health services in first 6 months of life. BMJ 1999 Jun 26;318:1740-4.

QUESTION: In mothers with infants $\leq 6$ months of age, does the distribution of the booklet Baby Check (an illness scoring system) affect their use of health services?

\section{Design}

Randomised (allocation not concealed*), blinded (practitioners), * controlled trial with 6-month follow-up.

\section{Setting}

13 practices (53 general practitioners, 4400-11000 patients per practice) in southeastern Glasgow, Scotland.

\section{Patients}

997 mothers (mean age 29 y, 45\% primiparous) who had babies (53\% boys, $95 \%$ full term) born during a period of 14 months. Participants were excluded if physicians thought that mothers or babies were too sick for inclusion or if mothers did not speak English. Only the first child of a multiple birth was included. Follow-up was $94 \%$.

\section{Intervention}

After stratification by practice, participants were allocated to the Baby Check group $(\mathrm{n}=497)$ or a control group $(\mathrm{n}=500)$. All mothers received a copy of an accident prevention leaflet (Play It Safe), and mothers in the intervention group received a copy of the booklet Baby Check, which includes information about how to use the Baby Check illness scoring system and when to consult a physician or health visitor.

\section{Main outcome measure}

Consultations with physicians were assessed by reviewing general practice notes.

\section{Main results}

No differences existed between groups for the use of primary care services $(p>0.2)$ (table). The study had $80 \%$ power to detect a $10 \%$ relative difference at a $5 \%$ level of significance. For both groups, 2 general practice consultations was the median rate during the first 6 months of life.

\section{Conclusion}

In mothers with infants $\leq 6$ months of age, the distribution of the booklet Baby Check did not affect frequency of use of health services.

*See glossary.

\section{COMMENTARY}

Deciding whether an infant is mildly or seriously ill can be difficult. Baby Check was developed to help parents and physicians assess the severity of acute illness in infants by scoring 19 symptoms and signs and to empower parents to get help for ill babies. ${ }^{1-4}$ It gives advice on whether an infant needs to be seen by a physician but does not give a diagnosis or management plan.

Thomson and colleagues did not educate mothers on how to use the list. The study assessed the use of health services and showed no difference between the groups.

We know little about why mothers consult a physician. This interesting study shows that most consultations for infants are for minor illnesses. This does not shed light on whether Baby Check would have empowered mothers of severely ill infants to obtain urgent and appropriate medical care. It also leaves many questions unanswered. Did the mothers who received Baby Check read and use it? Did they use it correctly? Did they think it helped them assess their babies' illnesses? Were the mothers' scores a true reflection of their babies' illnesses? Did the physicians think the mothers' concerns were appropriate?

Reports of the physicians' diagnoses and prescriptions are not relevant to whether Baby Check helped the mother assess her infant's illness. These only indicate the physicians opinion and not the accuracy of Baby Check. Studies have shown that physicians also have difficulty assessing severity of illness in infancy.

The trial by Thomson and colleagues assures us that mailing Baby Check does not alter the overall frequency of consultations. However, the question that really needs to be answered is whether the use of Baby Check by mothers and doctors means that seriously ill infants are recognised and treated earlier.

Colin Morley, MA, DCH, MD Royal Women's Hospital

Carlton, Victoria, Australia

1 Morley CJ, Thornton AJ, Cole TJ, et al. Baby Check: a scoring system to grade the severity of acute systemic illness in babies under 6 months old. Arch Dis Child 1991;66:100-5.

2 Morley CJ, Thornton AJ, Green SJ, et al. Field trials of the Baby Check score card in general practice. Arch Dis Child 1991;66:111-14.

3 Thornton AJ, Morley CJ, Cole TJ, et al. Field trials of the Baby Check score card in hospital. Arch Dis Child 1991;66:115-20.

4 Thornton AJ, Morley CJ, Green SJ, et al. Field trials of the Baby Check score card: mothers scoring their babies at home. Arch Dis Child 1991;66:106-10.

Source of funding. Chief Scientist Office of the Scottish Office Department of Health.

For correspondence: $\mathrm{Dr}$ $H$ Thomson, $M R C$ Social and Public Health Sciences Unit, 4 Lilybank Gardens, Glasgow G12 8RZ, UK. FAX

$+44-(0) 131-650-2681$

Baby Check booklet $v$ no booklet in mothers with infants $\leqslant 6$ months of aget

\begin{tabular}{lllll}
$\begin{array}{l}\text { Outcomes at } 6 \\
\text { months }\end{array}$ & Baby Check & Control & RBR $(95 \% \mathrm{Cl})$ & NNH \\
\hline 0 consultations & $17 \%$ & $19 \%$ & $11 \%(-17$ to 32$)$ & Not significant \\
\hline & & & $R R I(C l)$ & \\
\hline$\geqslant 5$ consultations & $22 \%$ & $19 \%$ & $17 \%(-9$ to 52$)$ & Not significant \\
\hline
\end{tabular}

†RBR = relative benefit reduction. Other abbreviations defined in glossary; RRI, NNH, and $\mathrm{Cl}$ calculated from data in article. 